\title{
A Comparison of Workspace and Force Capabilities between Classes of Underactuated Mechanisms
}

\author{
Ravi Balasubramanian and Aaron M. Dollar
}

\begin{abstract}
We propose a novel approach to study the ability of an underactuated mechanism, or a mechanism that has fewer actuators than degrees of freedom, to passively adapt to environmental constraints. While prior work in underactuated robotic hands has primarily focused on the mechanism's ability to curl its distal degrees of freedom inward even after the proximal degrees of freedom are constrained by contact with the environment, this paper explores the mechanism's adaptability in terms of both motion and force-application capabilities in the presence of external constraints. Specifically, using four different transmissions for a novel singly-actuated linear three degree-of-freedom mechanism, this paper analyzes how the system's ability to reconfigure joints and apply new contact forces varies as a function of the transmission configuration and object geometry. We show that with more extensive rerouting of a single actuator to multiple joints, the mechanism exhibits greater motion and force adaptability at the cost of decreased maximum joint travel and contact forces.
\end{abstract}

\section{INTRODUCTION}

Underactuated mechanisms are often employed in mechanical systems in order to allow for the passive accommodation of environmental constraints and disturbances while reducing sensing and hardware complexity. For example, underactuated differential transmissions are utilized on almost all automobiles in order to allow wheels driven by the same drive shaft to rotate at differing rates to accommodate for turning without loss of traction. In recent years, the robotics community has increasingly exploited the concept of underactuation in robotic devices. This is particularly true in the domain of robotic grasping and manipulation where an underactuated robotic hand's passive adaptability enables the hand to increase the number of links in contact with an object, thereby providing better control over the grasp [9], [3], [5], [14], [15], [12]. The key to these favorable characteristics in underactuated mechanisms is the underlying self-adaptive transmission that allows certain degrees of freedom to move even if another degree of freedom is locked due to external constraints.

The performance of underactuated mechanisms is a function of a number of parameters, many of which have been examined to some extent in previous work: kinematic configuration [3], [7], joint stiffnesses [7], coupling ratio [8], and actuation type [1]. However, the fundamental aspect of how the mechanism's transmission, which defines the coupling between the actuator and the degrees of freedom,

This work was supported in part by the National Science Foundation grant IIS-0953856. R. Balasubramanian and A. M. Dollar are with the Department of Mechanical Engineering at Yale University, New Haven, CT. \{ravi.balasubramanian, aaron.dollar\}@yale.edu. influences the mechanism's adaptability has yet to be examined. Specifically, the transmission determines the amount of force that is transmitted to each of the mechanism's joints. This paper investigates how an underactuated mechanism's adaptability (that is, its adaptive joint travel and forceapplication capability) varies with the transmission's force distribution and contact scenarios.

The majority of existing underactuated mechanisms described in the literature utilize revolute degrees of freedom in the plane. However, variations in behavior arising from the non-linear geometry of revolute mechanisms and different environmental contact scenarios (such as sliding and rolling over objects) greatly increase the complexity and parameter space and reduce the generality of the analysis of mechanism adaptability. To reduce the influence of these factors, we approach this problem using a novel linear underactuated system where the simple geometrical relationship between the mechanism's actuation and the degrees of freedom maintains the focus on the mechanism's motion and force adaptability.

We begin by providing a brief background on the underactuated mechanisms used commonly in the literature in section II, followed by a description of the linear underactuated system and possible transmissions for a singlyactuated mechanism in section III. We then present results in section IV from studies of the motion and force adaptability of the different transmission mechanisms. Finally, we discuss insights from these results and describe how they relate to planar revolute underactuated mechanisms in section V.

\section{BACKGROUND ON UNDERACTUATED MECHANISMS}

It is important to note the difference between underactuated mechanisms and fixed-motion coupling mechanisms. A fixed-motion coupled mechanism has more joints than degrees of freedom, with each degree of freedom controlled by a dedicated actuator. In these mechanisms, the motion of one joint always results in a proportional motion of the joint(s) coupled to it. Simultaneously, an external contact that constrains the motion of one joint also constrains all the coupled joints. Thus, fixed-motion joint coupling afford no passive motion adaptability [4], [13]. In contrast, underactuated mechanisms have fewer actuators than degrees of freedom and, therefore, demonstrate adaptive behavior through their unconstrained freedoms [9], [17], [11], [3]

Popular implementations of underactuation in robotic hands include cable-driven mechanisms and linkage-driven 


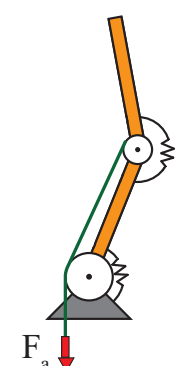

(a)

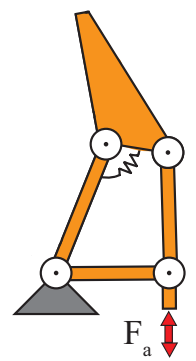

(b)
Fig. 1. Examples of underactuated mechanisms: a) a cable-driven system and (b) a linkage driven system.

mechanisms (see Fig. 1). In these mechanisms, the mechanism curls inward as the cable is pulled or the actuating link is pushed. Note that the proximal pulley in the cabledriven mechanism in Fig. 1a is a free-spinning pulley. In both these mechanisms, the distal links can continue to move even after contact occurs on the proximal links, allowing the mechanism to passively adapt to external constraints. This motion adaptability has been exploited in robotic grasping to produce a secure power grasp [6] where all the links make contact with the object.

Both the cable-driven and linkage-driven underactuated mechanisms transfer forces to the joints that are proportional to the actuator force $f_{a} \in \mathbb{R}$, and this relationship can be represented by $\left(\begin{array}{c}f_{a 1} \\ f_{a 2} \\ \vdots \\ f_{a n}\end{array}\right)=J_{a}^{T} f_{a}$, where $J_{a}=$ $\left(\begin{array}{llll}a_{1} & a_{2} & \ldots & a_{n}\end{array}\right) \in \mathbb{R}^{n}$ represents the mechanism transmission and $\left(\begin{array}{llll}f_{a 1} & f_{a 2} & \ldots & f_{a n}\end{array}\right)^{T} \in \mathbb{R}^{n}$ is the vector of joint-actuation generalized forces, and $n$ the number of degrees of freedom. Each element $a_{i} \in \mathbb{R}$ in $J_{a}$ represents the mechanism's transmission ratio for each degree of freedom. For example, $a_{i}$ is the pulley radius in cable-driven systems and the input-output ratio in linkage-driven mechanisms. Note that $J_{a}$ is a vector for the single-actuator system considered in this paper, but will have $n$ rows for a system with $n$ actuators.

In previous work on underactuated mechanisms, a mechanism's adaptability has loosely been referred to as its ability to move its distal joints after a proximal joint is externally constrained [9], [3]. However, adaptability also relates to the ability to produce different contact forces as the mechanism adapts to external constraints. So for the two-link underactuated finger, we instead describe the mechanism's "adaptability" as both the mechanism's ability to adjust the contact forces at the proximal and distal joints at various stages of the grasping process as well as its ability to move a joint after the other joint is constrained externally. Indeed, it is also important to move beyond this qualitative notion of adaptability and quantify it.

Interestingly, for a given transmission, an underactuated mechanism's adaptive behavior differs based on joint con- figuration, design parameters as well as the contact modes. For example, in most revolute joint configurations, external forces on the distal link of a cable-driven two-joint finger are transmitted to the proximal joint. However, if the distal link is perpendicular to the proximal link, then normal external forces do not produce any moments on the proximal joint. Also, if the pulley radii in the mechanism are equal and the distal link is locked by external constraints, then even a self-adaptive mechanism with multiple degrees of freedom mechanism is locked because of the singularity arising from actuation. Thus, in such special conditions, even an underactuated mechanism shows no adaptability. While some aspects of force and motion adaptability have been studied before for specific transmissions [3], the highly configuration-dependent behavior of serial-link chains with revolute joints and the huge parameter space (consisting of mechanism, configuration, transmission, and contact scenarios) makes them challenging to analyze.

\section{THE LINEAR UNDERACTUATED MECHANISM}

In order to focus on the fundamental differences between transmission schemes for an underactuated mechanism, we investigate a simpler, linear mechanism to explore the motion and force adaptability and workspace of an underactuated system as a function of the transmission and the contact conditions. Since the mechanism can only move along one dimension, the geometry of motion is significantly simpler than planar mechanisms that use revolute joints.

Figs. $2 \mathrm{a}$ and $2 \mathrm{~b}$ show a three degree-of-freedom linear singly-actuated mechanism, in this case actuated with two different tendon routings. Note that the different joint coupling schemes described in this paper can be achieved in many different ways including linkages and pneumatics, and each degree of freedom is a compliant prismatic joint. In the first transmission, the force is routed directly to the distal joint (see Fig. 2a), while in the second transmission mechanism, the force is routed to each joint individually (see Fig. 2b). The first transmission can be represented by a transmission matrix $J_{a}=\left(\begin{array}{lll}1 & 1 & 1\end{array}\right)$. Note that even though actuation is provided only to the distal joint, the force is transmitted to the proximal and distal joints through the links when the mechanism is unconstrained because the mechanism is linear.

The second transmission may be represented by the transmission matrix $J_{a}=\left(\begin{array}{lll}3 & 2 & 1\end{array}\right)$. When the mechanism is unconstrained, the net force seen at the proximal joint is the sum of the actuation forces provided at each joint. Since the actuation force provided to each joint is identical in the specific tendon-routing in Fig. $2 b$, the proximal joint experiences three times the force as the distal joint and the middle joint two times the force as the distal joint. Also the actuator force is divided by the pulleys between the various degrees of freedom in the second transmission. Indeed, only a quarter of the actuator force is provided directly to each joint.

The key point of showing these cable-routing mechanisms is that any transmission matrix can be achieved by carefully 


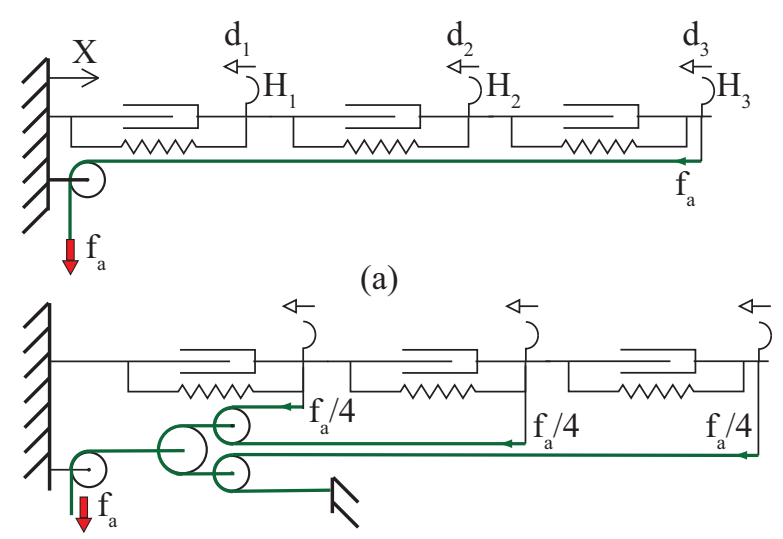

(b)

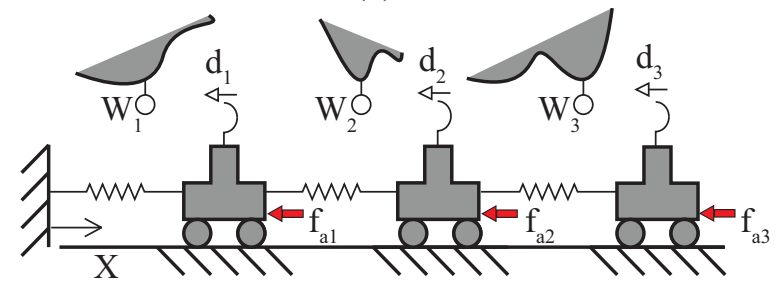

(c)

Fig. 2. A three degree-of-freedom linear underactuated mechanism driven by a single actuator: (a) A cable-driven system with actuation provided to the distal joint only; (b) A cable-driven system with actuation provided to all three joints; c) A schematic drawing of a linear underactuated mechanism with generalized joint actuation interacting with the environment. The whiteheaded arrows represent joint travel and the solid arrows forces.

exploiting tendons and pulleys or even other actuation systems such as pneumatics or gears. From here on, we will not restrict our discussion to cable-driven systems, and a jointactuation force will simply be represented by a force $f_{a i}$ at the joint without explicitly specifying how that force was created (see Fig. 2c). Thus, the joint-actuation forces $f_{a i}$ at joint $i$ in transmission 1 (see Fig. 2a) may be represented as $f_{a 1}=f_{a 2}=f_{a 3}=f_{a}$. On the other hand, the jointactuation forces $f_{a i}$ in transmission 2 (see Fig. 2b) may be represented as $f_{a 1}=3 f_{a} / 4, f_{a 2}=2 f_{a} / 4, f_{a 3}=f_{a} / 4$. For simplicity and without loss of generality, we will only consider the portion of the actuator force that is transmitted to the mechanism (disregarding the $f a / 4$ transferred to the wall). Then the joint actuation forces may be represented as $f_{a 1}=f_{a}, f_{a 2}=2 f_{a} / 3, f_{a 3}=f_{a} / 3$.

For a three degree-of-freedom linear mechanism with one actuator, the various canonical transmissions are provided in Table I. We only consider cases where the actuator has a nonzero effect on each degree of freedom and the distal joint is always actuated. Note that it is possible, at least in theory, to create a mechanism to achieve any transmission matrix $J_{a}$. However, the mechanism required may be complex. We have chosen configurations that we believe are intuitive and simple to implement.

With different transmission mechanisms, there are two ways to specify the maximum available joint-actuation force. The first option is to split the actuation force available to each joint depending on whether the actuation is double
TABLE I

Single-aCtuator TRANSmission CONFIGURATIONS

\begin{tabular}{|c|c|c|c|c|c|}
\hline & \multirow{2}{*}{\multicolumn{3}{|c|}{$\begin{array}{l}\text { Transmission } \\
\text { matrix } J_{a}\end{array}$}} & \multicolumn{2}{|c|}{ Maximum Force Per Joint } \\
\hline & & & & Unit force & Force normalization \\
\hline $\mathrm{AC}_{1}$ & 1 & 1 & 1 ) & 1 & 1 \\
\hline $\mathrm{AC}_{2}$ & 2 & 1 & 1 & 1 & 0.5 \\
\hline $\mathrm{AC}_{3}$ & 2 & 2 & $1)$ & 1 & 0.5 \\
\hline $\mathrm{AC}_{4}$ & 3 & 2 & $1)$ & 1 & 0.33 \\
\hline
\end{tabular}

routed $\left(\mathrm{AC}_{2}\right.$ and $\left.\mathrm{AC}_{3}\right)$ or triple routed $\left(\mathrm{AC}_{4}\right.$, see Fig. $\left.2 \mathrm{~b}\right)$. We will term this method "force normalization". The total actuator force available to the transmission system in the force-normalization convention is unity, and the maximum joint actuation forces available to each joint with force normalization are shown in Table I.

The second option is to provide unit force to each joint. This implies that the net force available from the actuator increases as the transmission routing becomes more complex. So the actuator force required for the $\mathrm{AC}_{4}$ transmission would be three times the actuator force required for the $\mathrm{AC}_{1}$ transmission. We will term this method "unit-force". Thus, a system with additional transmission routing will have greater joint-actuation forces than a system with a simpler transmission. We believe that the force-normalization convention offers a more realistic comparison of the various transmission systems. However, there are cases in which the unit-force approach might be of interest where multiple actuators are used to mimic the performance of a singleactuator routing system. Results of mechanism adaptability based on both conventions for specifying maximum jointactuation forces are provided in section IV.

In order to represent contact with the environment (without which underactuated mechanisms are uninteresting), the linear underactuated model includes "contact hooks" $H_{i}$, one for each degree of freedom, where it can interact with the environment, which also similarly has contact "hooks" $W_{i}$ (see Fig. 2). While various contact models such as compliant contact may be considered, this paper assumes that the contact point does not move once contact is made. An important benefit of underactuation is passive adaptability to unknown or unmodeled external objects, where the location of each environment contact hook $W_{i}$ is not known a priori. Thus, to ensure best performance and robustness to uncertainty, the mechanism must be designed for a variety of contact and contact-sequence possibilities. The ability of the transmission system to reconfigure to the various contact situations and apply forces at the contacts determines the system's adaptability.

\section{A. Contact Sequencing}

For a three degree-of-freedom mechanism, there are eight $\left(2^{3}\right)$ different possible contact modes. A fully-actuated mechanism can produce a full-dimensional set of forces in each of the contact states as well as move in any direction in the configuration space (subject to the operating contact constraints). However, an underactuated system's motion and force capabilities are more difficult to describe, since 


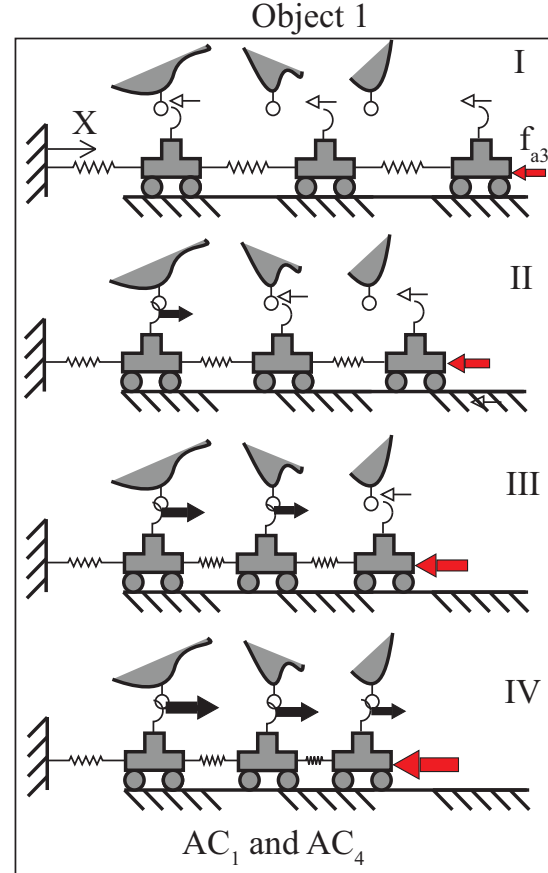

(a)

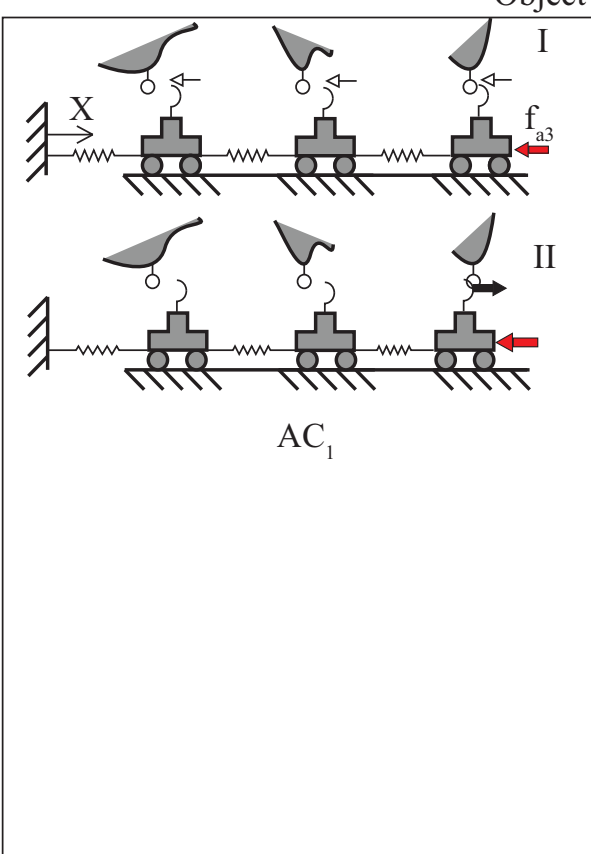

(b)

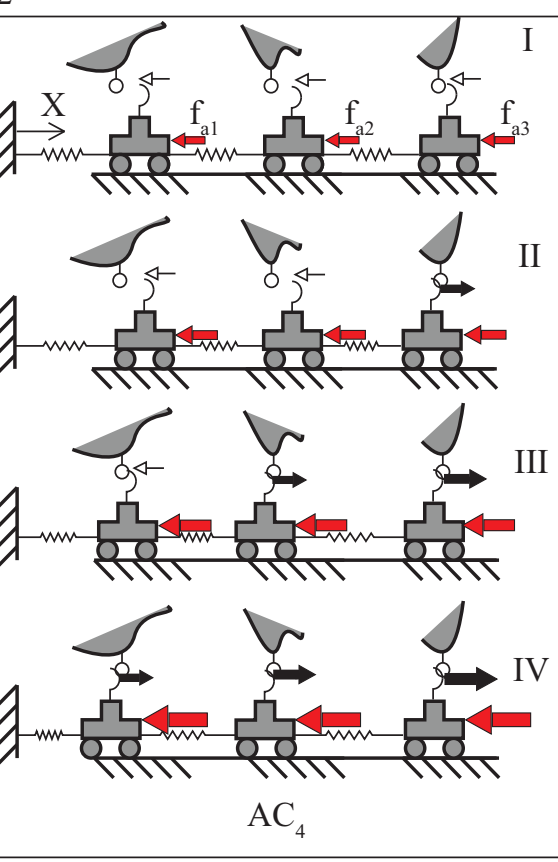

(c)

Fig. 3. Different grasping scenarios with the linear underactuated mechanism using transmissions $\mathrm{AC}_{1}$ and $\mathrm{AC}_{4}$. Using transmission $\mathrm{AC}_{1}$, a) object 1 's geometry results in every link making contact, b) while object 2's geometry results in only the distal links making contact. c) The additional actuation routing in $\mathrm{AC}_{4}$ enables the mechanism to adapt to both objects and make additional contacts.

the mechanism's joint actuation cannot be individually controlled and capabilities depend on mechanism design and contact constraints.

For a given transmission, the sequence of contacts is an important factor that determines how the underactuated mechanism moves and how the contact forces change. Indeed, even the simple linear mechanism, with eight contact possibilities, has twenty six unique contact sequences possible (no contact, three sequences leading to single contact, nine sequences leading to double-contact, and thirteen sequences leading to triple-contact). Each of these contact sequences results in different possibilities for joint travel and force application. We now explore these possibilities.

Consider a three degree-of-freedom system using transmission $\mathrm{AC}_{1}$. Since the actuation is routed only to the distal joint, a contact on the distal joint arising from appropriately located environmental hooks $W_{i}$ precludes the motion of more proximal joints (see Fig. 3b). However, Fig. 3a shows how the same transmission adapts to a contact sequence where contact first is made at the proximal joint, followed by contact at the middle joint, followed by contact at the distal joint. Indeed, most underactuated mechanisms are designed for such a contact sequence in order to produce a power grasp [9], [3]. However, it is difficult to predict how the grasp process evolves since object and robot hand locations and their "contact hooks" $\left(H_{i}\right.$ and $\left.W_{i}\right)$ are not known a priori. Thus, it is important to analyze how the mechanism performs across the entire space of different contact sequences in terms of motion and force capabilities to verify performance under varied conditions corresponding to different objects and grasp requirements.

Interestingly, the contact sequence in Fig. 3a can be visualized in the joint-travel space as well as the contactforce space (see Fig. 4a and Fig. 4b; section IIIC specifies how these spaces are computed). In the joint-travel space (see Fig. 4a), as the mechanism starts from rest, it moves from the origin along the vector OA. Once the proximal link makes contact, the mechanism "makes a turn" in the joint-travel space and moves along a vector parallel to line OB. Once the middle link has made contact, the mechanism again makes a turn in the joint-travel space and evolves along a vector parallel to line OC. The total travel by the mechanism is given by the addition of the three vectors traveled in the joint-travel space.

Three points are to be noted about the joint-travel space: 1) The net travel of a distal joint is the sum of its travel and the travel of the proximal joints. Thus, the net motion of the distal joint is $d_{1}+d_{2}+d_{3}$, and the distal joints typically travel greater lengths than the proximal joints. 2) The vectors OA, $\mathrm{OB}$, and $\mathrm{OC}$ in Fig. 4 represent unique directions in which the system can evolve in the displacement space. 3) The dashed lines $\mathrm{AB}, \mathrm{BC}$, and $\mathrm{CA}$ in Fig. $4 \mathrm{a}$ represent the limits of travel for the system when the actuator force is at its maximum, and the mechanism cannot travel along those lines.

Simultaneously, the system evolves in the force space also (see Fig. 4b). Before making any contacts, the system lies at the origin of the force space, since it does not apply 


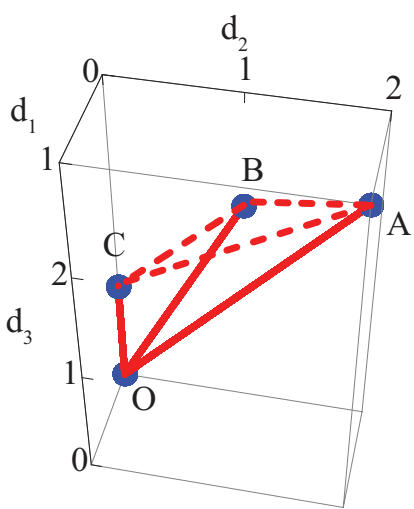

(a)

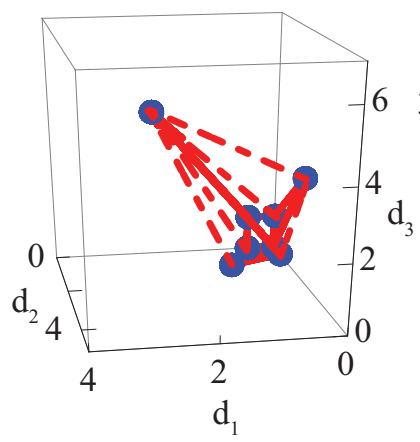

(c)

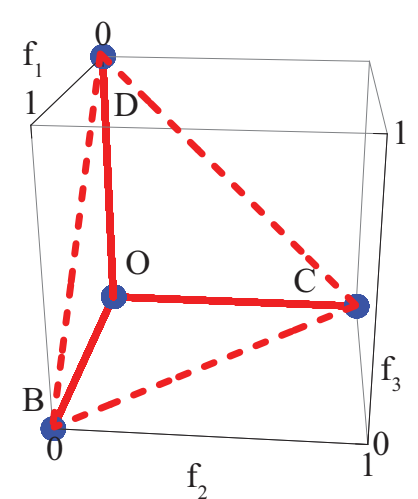

(b)

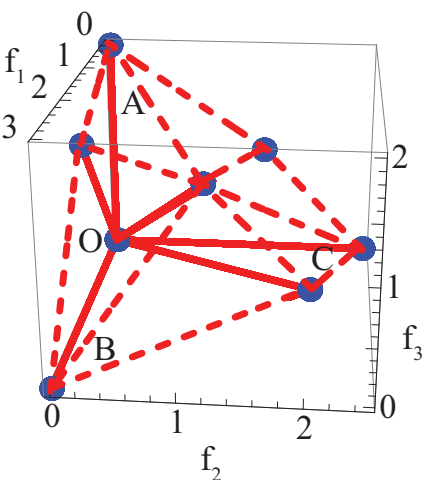

(d)
Fig. 4. Directions of travel in joint-travel (left column) and contactforce spaces (right column) for the linear underactuated mechanism with the $\mathrm{AC}_{1}$ (top row) and $\mathrm{AC}_{4}$ transmission (bottom row) using the unit-force convention. The axes in the joint-travel space represent joint-travel in spatial coordinates, while the axes in the contact-force space represent forces at each contact.

any forces. After making contact at the proximal link, the system evolves along the line OB. After making contact at the proximal link, the mechanism makes a turn in the force space and evolves along a direction parallel to the $\mathrm{OC}$ line. After making contact at the distal joint, the mechanism again makes a turn in the force space and evolves along a direction parallel to the OD line. The total force applied by the mechanism is given by the addition of the three vectors that the mechanism travels in contact-force space. This implies that the contact forces are maintained at the proximal joint even after distal contact is made.

While the above describes the performance of the $\mathrm{AC}_{1}$ transmission with the contact sequences depicted in Figs. $3 \mathrm{a}$ and $3 \mathrm{~b}$, the mechanism performance with, say, the $\mathrm{AC}_{4}$ transmission is significantly different. With the actuation being routed to all three joints in the $\mathrm{AC}_{4}$ transmission, the mechanism is not trapped by distal contact (see Fig. 3c), unlike the $\mathrm{AC}_{1}$ transmission (see Fig. 3b). Indeed, when moving in free space into a distal-contact state, the $\mathrm{AC}_{1}$ transmission is limited to travel only along OA in the jointtravel space and the vector OD in the contact-force space. In contrast, the $\mathrm{AC}_{4}$ mechanism can still reconfigure after the distal joint makes contact, and this ability can be represented by the additional unique directions the $\mathrm{AC}_{4}$ mechanism can travel in joint-travel space and force space (see Fig. 4c and Fig. 4d), when compared with the directions available to the $\mathrm{AC}_{1}$ mechanism.

\section{B. Adaptability}

The ability to reconfigure and modify the contact forces for any contact sequence is captured by the number of additional directions in which the system can evolve in the joint-travel space and the contact-force space beyond the directions available to the mechanism in the contact sequences that led to its current state. Table II shows the additional joint-travel and force directions available to the $\mathrm{AC}_{1}$ and $\mathrm{AC}_{4}$ transmissions for different contact sequences. For example, in the single-contact case, the $\mathrm{AC}_{1}$ transmission offers two new directions of joint-travel (beyond the nocontact situation) even after joints 1 and 2 are constrained. Specifically, the mechanism can travel along OB in the jointtravel space if contact is made on joint 1 and OC if contact is made on joint 2 (see Fig. 4a). But no travel is possible if contact is made on joint 3. In contrast, the $\mathrm{AC}_{4}$ system offers three new directions of travel in the single-contact state (beyond the no contact situation) even after any of the joints are constrained. In the contact-force space, both transmissions offer three new directions of force application for the single-contact case when compared with the nocontact situation, the directions corresponding to forces at the joints where contact occurs.

However, significant differences in joint-travel and forceapplication capabilities arise between the two transmissions for sequences that lead to double and triple contacts (see Table II). In the double-contact scenario, the motion capabilities depend on whether the mechanism transitioned into double-contact mode directly from the no-contact mode or evolved from single-contact mode to double-contact mode. For example, the $\mathrm{AC}_{1}$ transmission can only apply forces at the middle joint if it transitions directly from the nocontact mode to the contacts at the proximal and middle joint. However, if the $\mathrm{AC}_{1}$ transmission already had made contact at the proximal joint before making contact at the middle joint, it can apply forces at the middle joint in addition to the fixed force at the proximal contact. On the other hand, the $\mathrm{AC}_{4}$ transmission can increase forces at both the proximal joint and middle joint independent of which contact was made first. Indeed, the $\mathrm{AC}_{4}$ transmission has "more options" in joint-travel as well as force application even when adapting to the triple-contact mode (as shown in Table II).

This view of a mechanisms ability to reconfigure in joint space and apply different forces to the environment helps us define its adaptability. Indeed, as we notice from the above discussion, the extent of an underactuated mechanisms adaptability depends on the transmission as well as the contact sequence it experiences during operation. In this paper, we define adaptability of any contact sequence as the numbers of unique motions and force modifications available to it beyond the motions available in contact sequences that led to it. Then one possible definition of a transmission's 
TABLE II

Additional Travel Paths Available in Motion and Contact-Force Space for VARIOUS Contact SEQuences For the $\mathrm{AC}_{1}$ AND $\mathrm{AC}_{4}$ TRANSMISSIONS

\begin{tabular}{c|c|c|c|c}
\hline Contact sequence & \multicolumn{2}{|c|}{$\mathrm{AC}_{1}$} & \multicolumn{2}{c}{$\mathrm{AC}_{4}$} \\
& Motion & Force & Motion & Force \\
\hline None & 1 & 0 & 1 & 0 \\
None to Single & 2 & 3 & 3 & 3 \\
Single to Double & 1 & 3 & 6 & 6 \\
None to Double & 1 & 3 & 3 & 3 \\
Single to Double to Triple & 0 & 2 & 0 & 6 \\
Single to Triple & 0 & 2 & 0 & 3 \\
None to Double to Triple & 0 & 1 & 0 & 3 \\
None to Triple & 0 & 1 & 0 & 1 \\
\hline Total & 5 & 15 & 13 & 25 \\
\hline \hline
\end{tabular}

overall adaptability is the sum of all the numbers of unique motion and force modifications available to the system under all the possible contact sequences. From Table II, we see that the $\mathrm{AC}_{4}$ transmission, which has a joint-travel adaptability of thirteen and a contact-force adaptability of twenty five, is indeed more adaptable across all the contact modes than the $\mathrm{AC}_{1}$ transmission, which has a joint-travel adaptability of five and a contact-force adaptability of fifteen. Section IV provides details of the adaptability of all the four transmissions listed in Table I, and section V-A provides a discussion of how an understanding of the mechanism's adaptability can inform hand design.

\section{Static Analysis}

The linear underactuated mechanism's joint-travel and force-application capabilities after different contact sequences (the red vectors in Fig. 5) can be computed based on a statics analysis at each joint. The static balance at each contact state is given by

$$
J_{a}^{T} f_{a}+K d_{i}+J_{c}^{T} f_{i}=0,
$$

where $K \in R^{3 \times 3}$ represents mechanism's spring constants, $J_{c} \in R^{3 \times 3}$ the contact-constraint Jacobian, and $f_{i}$ the contact force at joint $i$. In this paper, we assume that the springs are linear and of unit magnitude even though they are tunable parameters. Note that the static equations (1) need to be solved in conjunction with the relevant contact constraints:

$$
\begin{array}{lc}
\text { Proximal contact } & d_{1}=0, \\
\text { Middle contact: } & d_{1}+d_{2}=0, \\
\text { Distal contact: } & d_{1}+d_{2}+d_{3}=0 .
\end{array}
$$

\section{RESULTS}

Considering the four transmissions with unit force provided to each joint shows that the maximum joint travel and contact forces the mechanism could apply increased from $\mathrm{AC}_{1}$ through $\mathrm{AC}_{4}$, since a mechanism with increased actuator routing uses larger actuator force.

However, the maximum joint-travel and contact forces for $\mathrm{AC}_{1}$ to $\mathrm{AC}_{4}$ with force normalization paints a different picture (see Fig. 5). Interestingly, joint travel for all joints and contact forces at all joints using $\mathrm{AC}_{1}$ is greater than

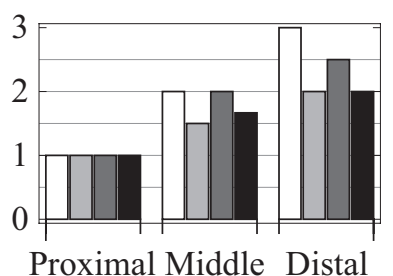

(a)

$\mathrm{AC}_{1}$ $\square \mathrm{AC}_{2}$

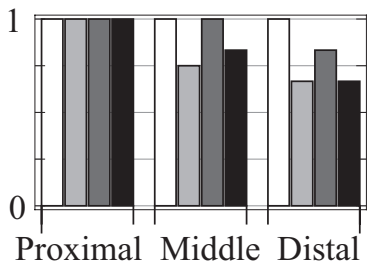

(b)
Fig. 5. (a) Joint-travel and (b) contact force limits for the four transmissions listed in Table I for the force-normalized system.

or equal to $\mathrm{AC}_{2}, \mathrm{AC}_{3}$ and $\mathrm{AC}_{4}$. This is because with force normalization, only a fraction of the actuation force is provided to the distal joint which reduces travel at those joints. Also, maximum middle-joint travel and contact forces using $\mathrm{AC}_{3}$ is larger than with $\mathrm{AC}_{2}$. This is expected since $\mathrm{AC}_{3}$ routes actuation to the middle joint, while $\mathrm{AC}_{2}$ routes actuation to joint 1 .

Fig. 6 shows the variation in motion adaptability of the linear underactuated mechanism for all four different transmissions according to the definition of adaptability provided in section IIIB. We notice that joint-travel adaptability increases as the mechanism moves from single to double to triple routing, allowing the mechanism to reconfigure to environmental constraints. Each of the bars contains four components, which detail the additional motions available after each contact sequence (the mechanism cannot move in the triple-contact mode) and the enumeration is similar to the example provided in Table II. We notice that $\mathrm{AC}_{2}$ provides more motion adaptability than $\mathrm{AC}_{3}$. This is because contact constraints at joint 2 reduce the motion adaptability of $\mathrm{AC}_{3}$ which double-routes actuation to joint 2 . In general, it was noticed that the more distal the routing, the greater the loss in adaptability due to contact constraints at that joint.

Fig. 7 shows the variation in force adaptability of the linear underactuated mechanism across the four different transmissions. Again, we notice that force adaptability increases as the mechanism moves from single to double to triple routing, allowing the mechanism to modify contact forces after different contact sequences. Each of the bars contain seven components, which detail the additional contact-force directions available after each contact sequence. The enumeration is similar to the example provided in Table II. Again, we notice that $\mathrm{AC}_{2}$ provides more force adaptability than $\mathrm{AC}_{3}$.

\section{DISCUSSION}

The linear underactuated mechanism offers interesting insights into how the transmission influences an underactuated system's performance. Specifically, it has shown that with more extensive re-routing of a single actuator to the joints with force normalization, the mechanism exhibits greater motion and force adaptability at the cost of decreased maximum joint-travel and contact forces (without considering the increased complexity of mechanism routing). 


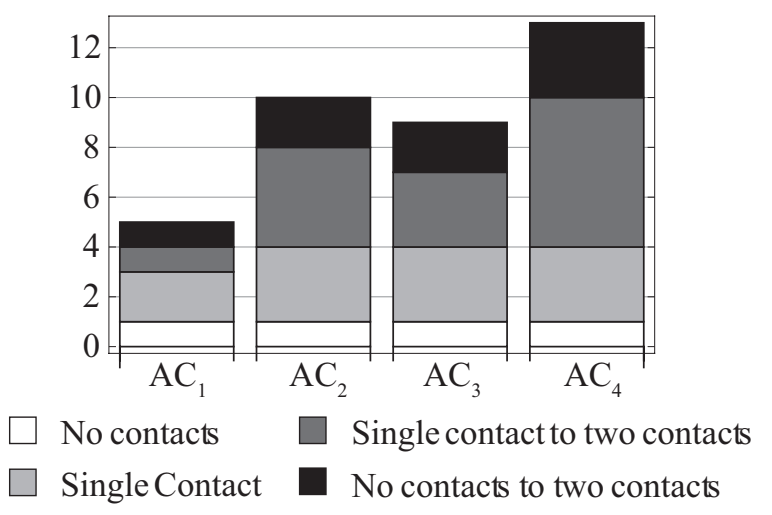

Fig. 6. Motion adaptability of the linear underactuated mechanism using the four transmissions listed in Table I.

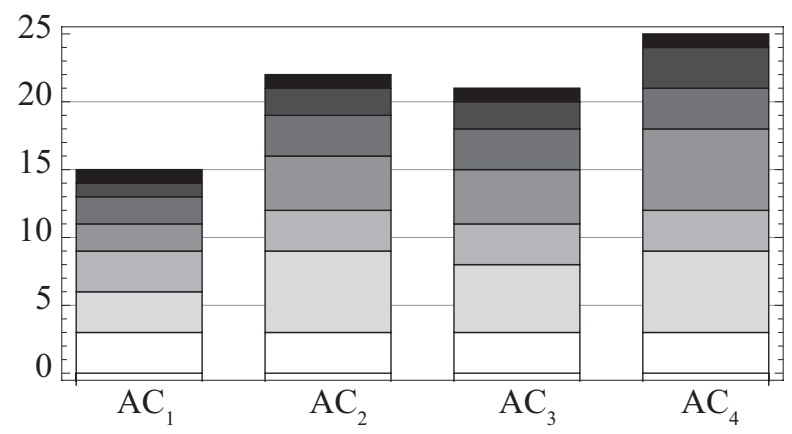

Single contact

Single contact to two contact

No contacts to double contacts

Single to double to triple contact

Single contact to triple contact

No contacts to double contact to triple contact

No contacts to triple contact

Fig. 7. Force adaptability of the linear underactuated mechanism using the four transmissions listed in Table I.

\section{A. Mechanism Design}

Mechanism design in robotics deals with the question of how to choose a mechanism's kinematics and actuation to achieve a specific motion and force capability. With fullyactuated systems, the design focus is predominantly on endeffector behavior. For example, to achieve pick-and-place tasks, the design effort is to ensure that the robot endeffector's workspace covers the required volume in threedimensional space and is able to produce the required forces to lift and transfer the object. However, the design problem for underactuated mechanisms is different, because the entire mechanism is an effector, much like whole-body manipulation that some researchers have explored with fullyactuated systems [10], [16]. In underactuated mechanisms, the beneficial passive adaptability comes at the expense of being unable to individually control the torques and joint motions.

The design process for underactuated-mechanisms hence must involve careful choice of the mechanism parameters and

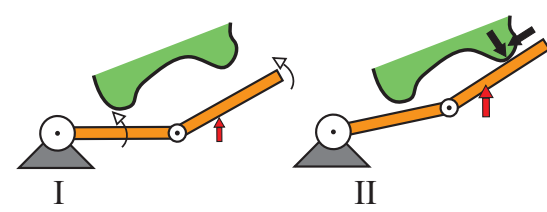

(a)

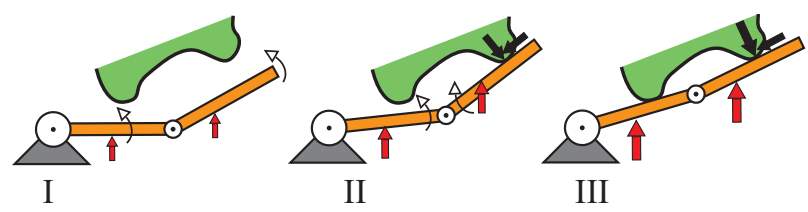

(b)

Fig. 8. Mechanism adaptability in a planar underactuated mechanism using transmissions for revolute mechanisms that are equivalent to the a) $\mathrm{AC}_{1}$ and b) $\mathrm{AC}_{4}$ transmissions described for the linear underactuated system. The two red arrows in (b) are coupled forces.

transmission scheme based on the desired contact forces and motions at various mechanism configurations and expected contact conditions. Most underactuated hands have been designed with the simple goal of distal joints continuing to wrap around even after the proximal links are constrained [9], [3]. Others have also designed underactuated mechanisms with the goal of a desired ratio of contact forces at the mechanisms links at specific joint configuration and assuming specific contact modes [12]. We propose through this paper that a more detailed exploration of a mechanisms motion and force adaptability is required to inform underactuated mechanism design. Our framework provides a way to find transmissions that will satisfy specific force and motion requirements by computing the additional directions of motion and forces that the mechanism can produce under different contact conditions.

Also, the transmission system used across a majority of existing underactuated systems are essentially similar to the one proposed many years ago [11]. We hope that the various transmission systems we explore through the linear model will provide inspiration for more novel transmission designs in underactuated mechanisms.

\section{B. Application of the Linear Underactuated Mechanism to Existing Systems}

An important aspect of the linear model is that it has only one dimension, and so a contact constraint on one joint can nullify the actuation force provided to another joint. A specific case is a distal-joint contact that can block the actuation forces to the proximal joints with the $\mathrm{AC}_{1}$ transmission (see Fig. 3b). However, the behavior of planar underactuated mechanisms that use revolute joints is different in this regard. Indeed, the contact Jacobian $J_{c}$ for planar mechanisms includes elements that couple planar forces with moments through the mechanism kinematics (as noted in section II).

Fig. 8a shows an example of a planar two-joint finger with a transmission for revolute mechanisms that is equivalent to $\mathrm{AC}_{1}$, while Fig. 8b shows a transmission equivalent to $\mathrm{AC}_{4}$. 
In the equivalent $\mathrm{AC}_{1}$ transmission, the actuation force (red solid arrow) is routed only to the distal joint, while in the equivalent $\mathrm{AC}_{4}$ transmission, the actuation is routed to both joints. Phase II in Fig. 8a indicates that there are certain joint configurations and contact conditions such that the $\mathrm{AC}_{1}$ mechanism is locked and the finger is limited to just a single contact with the object (the actuation force is balanced by the normal and frictional contact forces, resulting in zero reaction forces at joint 2 and moments about joint 1 ). In contrast, phases II and III in Fig. 8b indicate that the equivalent $\mathrm{AC}_{4}$ transmission can still reconfigure after making contact at the distal link and continue motion to establish contact with the object at the proximal link also. Such specific cases of reconfiguration have been noticed earlier in tendon-driven and linkage-driven underactuated mechanisms but only for specific transmissions [3], [2]. Furthermore, motion and force adaptability for revolute planar underactuated mechanisms are a strong function of the mechanism parameters as well as the contact scenario. In this paper, we sidestepped many of these important issues with the linear mechanism in order to focus on how the mechanisms adaptability varies with transmission. We will use the insights derived from the linear mechanism to further explore the adaptability of revolute underactuated mechanisms in the future.

\section{ACKNOWLEDGMENT}

The authors thank Joseph Belter for his helpful discussions related to this work.

\section{REFERENCES}

[1] R. Balasubramanian, J. T. Belter, and A. M. Dollar. External disturbances and coupling mechanisms in underactuated hands. In Proc. of ASME Internat. Design Engineering Technical Conf and Computers and Information in Engineering Conf., 2010.

[2] V. Begoc, C. Durand, A. Kurt, E. Dombre, and F. Pierrot. On the form-closure capability of robotic underactuated hands. In Proc. of the Internat. Conf. on Control, Automation, Robotics, and Vision, pages 2011-2018, 2006.
[3] L. Birglen, T. Laliberté, and C. Gosselin. Underactuated Robotic Hands. Springer, 2008.

[4] J. Butterfass, M. Grebenstein, H. Liu, and G. Hirzinger. DLR-Hand II: next generation of a dextrous robot hand. In Robotics and Automation, 2001. Proceedings 2001 ICRA. IEEE International Conference on, volume 1, pages 109-114 vol.1, 2001.

[5] R. M. Crowder, V. N. Dubey, P. H. Chappell, and D. R. Whatley. A multi-fingered end effector for unstructured environments. In Proc. IEEE Internat. Conf. on Robotics and Automation, pages 3038-3043, 1999.

[6] M. Cutkosky. On grasp choice, grasp models, and the design of hands for manufacturing tasks. Robotics and Automation, IEEE Transactions on, 5(3):269 -279, June 1989.

[7] A. M. Dollar and R. D. Howe. Towards grasping in unstructured environments: Grasper compliance and configuration optimization. Adv. Robotics, 19(5):523-544, 2005.

[8] A. M. Dollar and R. D. Howe. Joint coupling design of underactuated grippers. In Internat. Design Engineering Technical Conf and Computers and Information in Engineering Conf., pages 903-911, 2006.

[9] A. M. Dollar and R. D. Howe. The SDM Hand: A highly adaptive compliant grasper for unstructured environments. Internat. J. Robotics Res, 2010.

[10] K. Harada and M. Kaneko. Whole body manipulation. In Robotics, Intelligent Systems and Signal Processing, 2003. Proceedings. 2003 IEEE International Conference on, volume 1, pages 190 - 195 vol.1, oct. 2003.

[11] S. Hirose and Y. Umetani. The development of soft gripper for the versatile robot hand. Mechanism and IVlechine Theory, 13:351-359, 1978.

[12] Y. Kamikawa and T. Maeno. Underactuated five-finger prosthetic hand inspired by grasping force distribution of humans. In Intelligent Robots and Systems, 2008. IROS 2008. IEEE/RSJ International Conference on, pages $717-722$, sep. 2008.

[13] C. Lovchik and M. Diftler. The robonaut hand: a dexterous robot hand for space. In Robotics and Automation, 1999. Proceedings. 1999 IEEE International Conference on, volume 2, pages 907-912 vol.2, 1999.

[14] M. Rakic. Multifingered hand with self-adaptability. Robotics and Computer-Integrated Manufact., 3(2):269-276, 1989.

[15] A. Rovetta. On functionality of a new mechanical hand. ASME J. Mechanical Design, 103:277-280, 1981.

[16] M. Stilman, K. Nishiwaki, and S. Kagami. Humanoid teleoperation for whole body manipulation. In Robotics and Automation, 2008. ICRA 2008. IEEE International Conference on, pages $3175-3180$, may. 2008.

[17] N. Ulrich and V. Kumar. Grasping using fingers with coupled joints. In In Proc. ASME Mechanisms Conference, 1988. 